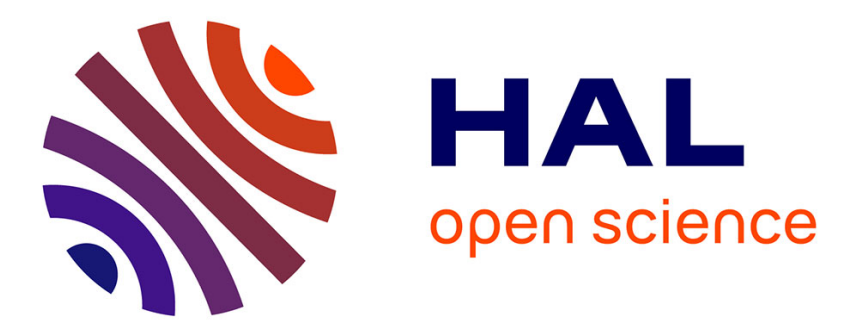

\title{
Dielectric properties of a single nanochannel investigated by high-frequency impedance spectroscopy
}

Jean Gamby, François-Damien Delapierre, Antoine Pallandre, Bernard

Tribollet, Claude Deslouis, Anne-Marie Haghiri-Gosnet

\section{- To cite this version:}

Jean Gamby, François-Damien Delapierre, Antoine Pallandre, Bernard Tribollet, Claude Deslouis, et al.. Dielectric properties of a single nanochannel investigated by high-frequency impedance spectroscopy. Electrochemistry Communications, 2016, 66, pp.5-9. 10.1016/j.elecom.2016.02.011 . hal01297990

\section{HAL Id: hal-01297990 \\ https://hal.sorbonne-universite.fr/hal-01297990}

Submitted on 7 Apr 2016

HAL is a multi-disciplinary open access archive for the deposit and dissemination of scientific research documents, whether they are published or not. The documents may come from teaching and research institutions in France or abroad, or from public or private research centers.
L'archive ouverte pluridisciplinaire HAL, est destinée au dépôt et à la diffusion de documents scientifiques de niveau recherche, publiés ou non, émanant des établissements d'enseignement et de recherche français ou étrangers, des laboratoires publics ou privés. 


\title{
Dielectric properties of a single nanochannel investigated by high frequency impedance spectroscopy
}

\author{
Jean Gamby, ${ }^{\mathrm{a}, \mathrm{b}, \dagger}$ François-Damien Delapierre, ${ }^{\mathrm{b}}$ Antoine Pallandre, ${ }^{\mathrm{b}, \mathrm{c}}$ Bernard Tribollet, ${ }^{\mathrm{a}}$ \\ Claude Deslouis, ${ }^{\mathrm{a}}$ and Anne-Marie Haghiri-Gosnet ${ }^{\mathrm{b}}$ \\ ${ }^{a}$ Sorbonne Universités, UPMC Univ Paris 06, CNRS, Laboratoire Interfaces et Systèmes \\ Electrochimiques, 4 place Jussieu, F-75005, Paris, France \\ ${ }^{b}$ CNRS, Laboratoire de Photonique et de Nanostructures, UPR20, Marcoussis 91460, France \\ ${ }^{c}$ Univ Paris-Sud, Faculté de Pharmacie, 5 rue Jean-Baptiste Clément, F-92 296, Châtenay- \\ Malabry, France
}

${ }^{\dagger}$ Corresponding author: jean.gamby@upmc.fr Tel: +33 14427 36 40; Fax: +33 14427 4074; 


\begin{abstract}
The high frequency electric impedance spectroscopy is used to characterize the dielectric properties of a single nanochannel in a micro/nanofluidic device. The simulated electric impedance results lead to the determination of two conductance regimes, a non ideal capacitance and its surface charge.
\end{abstract}

Keywords: nanochannel; impedance, high frequency, conductance, constant phase element, surface charge. 


\section{Introduction}

The understanding of the physics of ionic transport in biologic membranes, solid-state nanofluidic devices or porous material offers new perspectives from biology to energy storage domains. [1],[2],[3],[4],[5],[6],[7],[8],[9] In such systems characterized by at least one nanometer dimension, the role of the surface charge is exacerbated,[10],[11] and new phenomena such as surface-charge mediated transport and permselectivity occur. The unbalanced ionic transport between anionic and cationic species through a nanofluidic structures initiates a polarization effect related to the concentration domain, which can be applied for focusing and locally preconcentrate ionic species.[12],[13] Compared to the classical microfluidic field, the nanometer dimension of the channel requires specific theoretical predictions and experimental discussions.[14],[15],[16],[17]. The conductance law through nanochannels depends on the ionic strength of the background electrolyte.[18],[19],[20]. At high concentration for which the Debye length is much smaller than the channel height, conductance through the nanochannel follows the solution conductivity; at low concentration corresponding to a large Debye length with respect to the channel height, the nanochannel conductance becomes concentration independent and is fully governed by surface charge. Recently, Reed et. al. [20] have investigated the conductance change in an intermediate regime of ionic strength and have speculated about a surface charge inversion in divalent systems where ion-ion correlations exist. In this

context, development of direct real-time measurement of conductance in nanofluidic channel is of great interest and necessitates new strategies for more accurate characterization. 
In this letter, a new methodology to determine accurately the nanochannel electric properties in a micro/nano/microfluidic device using the high frequency (HF) electric impedance spectroscopy is presented. Indeed, the main limitation encountered with the use of traditional commercially available EIS instrument is the HF measurement accuracy. To overcome this limitation, we combined a HF EIS Frequency Analyzer with a dielectric interface apparatus dedicated to electric impedance measurements for high resistive materials.[21],[22],[23]

\section{Experimental}

\subsection{Chemicals and reagents}

$\mathrm{KCl}$ and $\mathrm{NaOH}$ (from Sigma-Aldrich) were used as background electrolyte solution and as washing solution for the glass surface regeneration, respectively.

\subsection{Nanochannel and microchannel fabrication}

The hybrid micro/nanofluidic device displayed in Fig. 1 was fabricated in borosilicate glass chip with a $1.47 \pm 0.01 \mu \mathrm{m}$ height microchannel and a $170 \pm 10 \mathrm{~nm}$ height nanochannel. Nanochannel was made using the following process. 1) First, $300 \mathrm{~nm}$ of amorphous silicon $(\alpha-\mathrm{Si})$ was deposited by plasma-enhanced chemical vapor deposition on a $500 \mu \mathrm{m}$ glass wafer. 2) Then AZ-5214 reversible photoresist was spin-coated (5000 rpm, $30 \mathrm{~s}$ ) on the $\alpha$ Si glass substrate. 3) The microchannels were designed by UV lithography on a SUSS MicroTec MJB4 mask aligner. 4) In the regions that were not protected by the resin, the $\alpha$ $\mathrm{Si}$ was etched by reactive-ion etching (RIE) with $\mathrm{SF}_{6}$. 5) The microchannels were then etched, on $\alpha$-Si not protected areas of the glass, using ammonium fluoride - hydrofluoric acid mixture (AF 875-125, etching mixture, Aldrich). 6-9) The nanochannel was made by 
the same method after a second spin-coating, lithography and RIE $\left(\mathrm{SF}_{6}\right)$ procedure. 10) The reservoirs, where solutions were injected, were drilled by sandblasting. 11) The remaining $\alpha$-Si was removed using RIE $\left.\left(\mathrm{SF}_{6}\right) .12\right)$ The glass chip and another $300 \mu \mathrm{m}$ glass wafer were then carefully washed using, successively, trichloroethylene, acetone and isopropyl alcohol, the etching mixture for a few seconds, and deionized water.. 13) The two wafers were bonded without any intermediate layer using a wafer bonder SB6 (SUSS MicroTec). During thermal bonding, wafers were put in contact for $2 \mathrm{~h}$ under vacuum at $550^{\circ} \mathrm{C}$ with an applied pressure of 3-5 bars. 14) A 5 mm layer of polydimethylsiloxane (PDMS) with holes aligned with the reservoirs was bonded on the chip using air plasma for $70 \mathrm{~s}$.

- Fig. 1 -

\subsection{Instrumentation and measurement}

Electric impedance measurements were performed with a frequency response analyser (FRA 1255B) coupled with a dielectric interface (DI 1296A) which extends frequency range up to $20 \mathrm{MHz}$. The originality derives from the use of this latter instrument which contains a very high input impedance allowing measurement of ultralow currents. [24],[25] Impedance was measured between two $\mathrm{Ag} \mid \mathrm{AgCl}$ junctions located in the microchannel reservoirs (Fig. 1). The frequency range was varied from $1 \mathrm{MHz}$ to $0.1 \mathrm{~Hz}$. The sinusoidal ac signal amplitude was set to $100 \mathrm{mV}$, and no dc bias was imposed in order to avoid any faradic reactions.

\section{Results and discussion}

3.1. Electric impedance spectroscopy on micro nanodevice 
To determine the nanochannel dielectric properties, the procedure described below was followed for various $\mathrm{KCl}$ concentrations. Solutions were introduced by capillarity in the device and measurements started only after a stabilization time of 45 minutes in order to reach the steady state conditions for complete solution uptake through the nanochannel. The results are displayed according the Nyquist or Bode (modified as phase and log (imaginary) vs. $\log$ (f)) plot representations. As displayed in Fig. $2 \mathrm{~A}-\mathrm{B}$ for $3 \times 10^{-4} \mathrm{M} \mathrm{KCl}$ concentration, characteristic frequencies are indicated on the plot to visualize the time constants of phenomena involved. Indeed, the semi-circle shape suggests a traditional $R / / C$ electrical circuit with a characteristic frequency $f_{\mathrm{RC}}$ that can be also identified on the $Z_{\mathrm{j}}$ and phase Bode diagrams. This $f_{\mathrm{RC}}$ value, equal to $1168 \mathrm{~Hz}$, corresponds to the maximum of $Z_{\mathrm{j}}$, equal to $R / 2$ ( $R$, semi-circle diameter). Furthermore, the nanochannel resistance, $R$, can be estimated as the difference on the real-axis between the asymptotic resistance limit at zero-frequency, $R_{\mathrm{LF}}$, and that as frequency tends towards $\infty, R_{\mathrm{HF}}$, (Fig. 2A-B). As one can observe on real-axis, $R_{\mathrm{HF}}$, is negligible, then $R$ identifies itself with $R_{L F}$. It is observed that the nanochannel capacitance, $C$, obtained in the HF domain is not ideal $\left(\varphi \neq 90^{\circ}\right)$ because the impedance phase tends towards a constant value equals to $-88^{\circ}$ (Fig. 2B). This experimental result is also confirmed since the $\alpha$-slope value for $Z_{\mathrm{j}}$ with frequency on log$\log$ scale indicated in Fig. $2 \mathrm{~B}$ is equal to $-0.98\left(\alpha=-88^{\circ} / 90^{\circ} \approx-0.98\right.$ thus different from the unit). In brief, it had been assumed that nanochannel capacity was a non ideal capacity because it is not independent of frequency. Indeed, this analysis of the data showed that it decreased with increasing frequency. Consequently, the glass/ nanochannel interface behaves as a $R C$ filter where a non ideal capacitor can be theoretically represented by a constant phase element (CPE). The changes on the effective CPE coefficient, $Q$, can be 
calculated from the obtained raw data of the imaginary part in the HF domain, as proposed by Orazem et. al.[26] with equation 1 as follows

$$
Q=\sin \left(\frac{\alpha \pi}{2}\right) \frac{-1}{Z_{j}(f)(2 \pi f)^{\alpha}}
$$

with $Q$ and $\alpha$ being the $\mathrm{CPE}$ element and the CPE exponent, respectively.

- Fig. 2 -

In equation 1 , when $\alpha=1$, the effective CPE coefficient, $Q_{\text {eff }}$, becomes an effective capacitance, $C_{\text {eff. }}$ The change of $Q$ was plotted by using $\alpha$-slope numerical value previously determined $(\alpha=-0.98)$ and the $Z_{\mathrm{j}}$ data at each frequency. As expected an asymptotic value $Q_{\text {lim }}=1.08 \times 10^{-12} \mathrm{~F} \mathrm{~s}^{(\alpha-1)}$ was deduced by plotting the $Q_{\text {eff variation that behaves as a CPE in }}$ the HF domain (Fig. 2C). At this stage, it can be assumed that the observed CPE behavior originates probably from the nanochannel geometry which induces a non uniform current and potential distribution at the nanochannel entrance with a CPE exponent, $\alpha$, taking into account the role of concentration polarization.

For exploring changes on conductance and capacitance, several $\mathrm{KCl}$ solutions were successively introduced from low to high concentrations $\left(3 \times 10^{-6} \mathrm{M}-3 \mathrm{M}\right)$. The data analysis was performed with a homemade software (Simad) using the electrical equivalent circuit displayed in Fig. 3A that corresponds to an analytical expression taking into account a CPE coefficient $Q$ instead of pure capacitor $C$, as follows

$$
Z(\omega)=R_{H F}+\frac{R}{1+(j \omega)^{\alpha} Q \cdot R}
$$


where $\omega$ is the angular frequency $\left(\operatorname{rad~s}^{-1}\right)$, defined by $\omega=2 \pi f$, and $f$ is the frequency $(\mathrm{Hz})$. As shown in Fig. 3B-C, the nanochannel resistance, $R$, decreases when the nanochannel ionic concentration increases from $3 \times 10^{-6}$ to $3 \mathrm{M}$. The corresponding conductance $R^{-1}\left(\Omega^{-1}\right)$ or $G(\mathrm{~S})$ vs ionic concentration is plotted in the log-log scale (see Fig. 3D). Two conductance regimes are highlighted: a plateau conductance appears for concentrations lower than $3 \times 10^{-2} \mathrm{~mol} \mathrm{~m}^{-3}$, while a linear conductance variation starts from concentrations higher than $30 \mathrm{~mol} \mathrm{~m}^{-3}$. These conductance regimes in a nanochannel have been also observed in the literature, using other methods such as dc conductance,[18] or by conventional electrochemical impedance spectroscopy measurements.[27] Indeed, as proposed in literature [18],[27], the conductance of a nanochannel filled with a 1:1 background electrolyte, in the steady-state conditions, neglecting electroosmotic effects, can be expressed as the sum of the bulk conductance (nanochannel opened), first term in equation 3, and the conductance due to the excess of counterions equilibrating surface charges (nanochannel closed), second term in equation 3 , as follows

$$
R^{-1}=G=2 \mu_{ \pm} c_{i} \cdot N_{A} \cdot e \frac{w h}{l}+2 \mu_{+} \frac{\sigma_{0} w}{l}
$$

where $\mu_{ \pm}$and $\mu_{+}$correspond to the average value of the ionic mobility and the positive charge mobility, respectively. $c_{i}$ represents the ionic concentration, $N_{\mathrm{A}}$ the Avogadro number, $e$ the electron charge. $w, h$ and $l$ are the nanochannel width, height and length, respectively. $\sigma_{0}$ is the effective surface charge density in the diffuse layer assuming that the species are immobile in the inner Helmholtz plane (IHP).

In the configuration here described the nanochannel width and length are identical leading to a simplified equation 3 as follows 


$$
G=2 \mu_{ \pm} c_{i} \cdot F \cdot h+2 \mu_{+} \sigma_{0}
$$

where $F$ is the Faraday constant.

A transition concentration, $\mathrm{c}_{t}$, delimiting these two regimes is obtained after equating the first and the second term, as follows

$$
c_{t}=\frac{\mu_{+} \sigma_{0}}{\mu_{ \pm} \cdot F \cdot h}=t_{+} \cdot \frac{\sigma_{0}}{F \cdot h}
$$

where the role of mobility ratio $t_{+}$(cationic transport number) is highlighted.

For the simulated conductance curve, we obtained an effective surface charge density value, $\sigma_{0}=-2.5 \times 10^{-2} \mathrm{C} \mathrm{m}^{-2}$ that is closely similar to those measured in the literature.[18],[28] In addition, $c_{t}$ graphically determined around $2.6 \mathrm{~mol} \mathrm{~m}^{-3}$ is in good agreement with the one estimated i.e. $3.1 \mathrm{~mol} \mathrm{~m}^{-3}\left(t_{+}=0.98\right.$ in equation 5).

- Fig. 3-

As displayed in Fig. 3D, it is obvious that the deviation to the electrical neutrality in the double layer (second term in equation 4) becomes qualitatively predominant compared to the average ionic strength at low concentrations. For the two lower concentrations, the Debye length is of the order of magnitude of the nanochannel height.[29] We also observe that the non ideal capacitance obtained does not depend on $\mathrm{KCl}$ concentration, confirming that it is an intrinsic parameter (a geometric capacity : $C=\left(\varepsilon_{\mathrm{R}} \varepsilon_{0} w l\right) / h$ ) would be more modulable by increasing the length of the nanochannel, $l$, and not its section $h$. In future work, we will investigate on the transition zone where a well-established phenomenon such as pre-concentration depends on the ionic strength of the background electrolyte. [20] 


\section{Conclusions}

We demonstrated that HF electric impedance spectroscopy can be used to determine nanochannel dielectric properties in a nanofluidic device. Modelling the obtained electric impedance data highlighted the existence of a linear variation of the nanochannel conductance for higher ionic concentrations, and of a plateau conductance for lower concentrations. A nanochannel conductance, surface charge, and non ideal capacitance were determined equal to $8.33 \mathrm{nS},-25 \mathrm{mC} \mathrm{m}^{-2}$ and $1.08 \mathrm{pF} \mathrm{s}^{\alpha-1}$, respectively.

\section{Conflict of interest}

The authors declare that they have no conflicts of interests. 


\section{References}

[1] E. Neher, B. Sakmann, Single-channel currents recorded from membrane of denervated frog muscle fibres, Nature, 260 (1976) 799-802.

[2] P. Abgrall, N.T. Nguyen, Nanofluidic Devices and Their Applications, Anal. Chem. (Washington, DC, U. S.), 80 (2008) 2326-2341.

[3] J.C.T. Eijkel, A. van den Berg, Nanofluidics: what is it and what can we expect from it?, Microfluid. Nanofluid., 1 (2005) 249-267.

[4] H. Daiguji, Y. Oka, T. Adachi, K. Shirono, Theoretical study on the efficiency of nanofluidic batteries, Electrochemistry Communications, 8 (2006) 1796-1800.

[5] R.B. Schoch, J. Han, P. Renaud, Transport phenomena in nanofluidics, Rev. Mod. Phys., 80 (2008) 839-883.

[6] S. Tseng, C.-H. Chang, J.-P. Hsu, B.-T. Liu, Influence of temperature on the electroosmotic flow in a pH-regulated, zwitterionic cylindrical pore filled with multiple monovalent ions, Electrochemistry Communications, 48 (2014) 169-172.

[7] L. Mei, L.-H. Yeh, S. Qian, Buffer effect on the ionic conductance in a pH-regulated nanochannel, Electrochemistry Communications, 51 (2015) 129-132.

[8] J. Gamby, P.L. Taberna, P. Simon, J.F. Fauvarque, M. Chesneau, Studies and characterisations of various activated carbons used for carbon/carbon supercapacitors, J. Power Sources, 101 (2001) 109-116.

[9] C. Pean, B. Daffos, B. Rotenberg, P. Levitz, M. Haefele, P.-L. Taberna, P. Simon, M. Salanne, Confinement, Desolvation, And Electrosorption Effects on the Diffusion of Ions in Nanoporous Carbon Electrodes, Journal of the American Chemical Society, 137 (2015) 12627-12632.

[10] Z. Yuan, A.L. Garcia, G.P. Lopez, D.N. Petsev, Electrokinetic transport and separations in fluidic nanochannels, Electrophoresis, 28 (2007) 595-610.

[11] H.-L. Gao, H. Zhang, C.-Y. Li, X.-H. Xia, Confinement effect of protonation/deprotonation of carboxylic group modified in nanochannel, Electrochimica Acta, 110 (2013) 159-163.

[12] A. Plecis, C. Nanteuil, A.-M. Haghiri-Gosnet, Y. Chen, Electropreconcentration with Charge-Selective Nanochannels, Anal. Chem. (Washington, DC, U. S.), 80 (2008) 95429550.

[13] A.-C. Louër, A. Plecis, A. Pallandre, J.-C. Galas, A. Estevez-Torres, A.-M. HaghiriGosnet, Pressure-Assisted Selective Preconcentration in a Straight Nanochannel, Analytical Chemistry, 85 (2013) 7948-7956.

[14] X. Xuan, D. Li, Solute separation in nanofluidic channels: pressure-driven or electric field-driven?, Electrophoresis, 28 (2007) 627-634.

[15] A. Garai, S. Chakraborty, Steric effect and slip-modulated energy transfer in narrow fluidic channels with finite aspect ratios, Electrophoresis, 31 (2010) 843-849.

[16] S. Movahed, D. Li, Electrokinetic transport through nanochannels, Electrophoresis, 32 (2011) 1259-1267.

[17] A.T. Conlisk, The Debye-Huckel approximation: Its use in describing electroosmotic flow in micro- and nanochannels, Electrophoresis, 26 (2005) 1896-1912.

[18] D. Stein, M. Kruithof, C. Dekker, Surface-Charge-Governed Ion Transport in Nanofluidic Channels, Phys. Rev. Lett., 93 (2004) 035901.

[19] I. Vlassiouk, S. Smirnov, Z. Siwy, Ionic Selectivity of Single Nanochannels, Nano Lett., 8 (2008) 1978-1985. 
[20] S.X. Li, W. Guan, B. Weiner, M.A. Reed, Direct Observation of Charge Inversion in Divalent Nanofluidic Devices, Nano Lett., 15 (2015) 5046-5051.

[21] J. Gamby, J.-P. Abid, H.H. Girault, Supercapacitive Admittance Tomoscopy, J. Am. Chem. Soc., 127 (2005) 13300-13304.

[22] M. Kechadi, J. Gamby, L. Chaal, H. Girault, B. Saidani, B. Tribollet, Polymer microchip impedance spectroscopy through two parallel planar embedded microelectrodes: Understanding the impedance contribution of the surrounding polymer on the measurement accuracy, Electrochimica Acta, 105 (2013) 7-14.

[23] M. Faure, B. Sotta, J. Gamby, Investigating the kinetics of paramagnetic-beads linked alkaline phosphatase enzyme through microchannel resistance measurement in dielectric microchip, Biosens Bioelectron, 58C (2014) 61-67.

[24] M. Kechadi, L. Chaal, B. Tribollet, J. Gamby, Dynamics of BSA adsorption onto a photoablated polymer surface in a dielectric microchip, Analyst (Cambridge, U. K.), 139 (2014) 1492-1497.

[25] M. Kechadi, B. Sotta, L. Chaal, B. Tribollet, J. Gamby, A real time affinity biosensor on an insulated polymer using electric impedance spectroscopy in dielectric microchips, Analyst (Cambridge, U. K.), 139 (2014) 3115-3121.

[26] M.E. Orazem, N. Pébère, B. Tribollet, Enhanced Graphical Representation of Electrochemical Impedance Data, Journal of The Electrochemical Society, 153 (2006) 129136.

[27] R.B. Schoch, H. van Lintel, P. Renaud, Effect of the surface charge on ion transport through nanoslits, Phys. Fluids, 17 (2005) 100604.

[28] R.B. Schoch, P. Renaud, Ion transport through nanoslits dominated by the effective surface charge, Appl. Phys. Lett., 86 (2005) 253111.

[29] M.-J. Huang, L. Mei, L.-H. Yeh, S. Qian, pH-Regulated nanopore conductance with overlapped electric double layers, Electrochemistry Communications, 55 (2015) 60-63. 


\section{Figure captions}

Fig. 1: Left : Global view of a microfluidic chip. At each corner, a hole is visible, where electrolyte is injected. Conductance changes between the two $\mathrm{Ag} \mid \mathrm{AgCl}$ junctions are measured either between holes 1 and 3 or between 2 and 4 . The nanochannel is in the middle, framed with blue solid line. Right : A schematic view of the micro/nano/micrometric geometry of the device in the middle. The microchannel is reduced to nanometric size on a short distance.

Fig. 2: A. Nyquist representation of electric impedance data of 170-nm high nanochannel measured in a $3 \times 10^{-4} \mathrm{M} \mathrm{KCl}$ electrolyte solution for frequencies from $1 \mathrm{MHz}$ to $0.1 \mathrm{~Hz}$. The diameter loop allows a quick estimation of $R \approx 134.9 \mathrm{M} \Omega$ for the nanochannel resistance. B. Bode representation of the electric impedance imaginary part (right-axis) superimposed with the Bode representation of the electric impedance phase (left-axis), underlining the inflexion point $\left(-45^{\circ}\right.$ phase $)$ when the maximum frequency $\left(f_{\mathrm{RC}}=1168 \mathrm{~Hz}\right)$ is reached. C. Bode representation of the effective CPE coefficient defined in equation 1, underlining a plateau obtention in the high-frequency asymptote, $Q_{\text {lim }}$, where an accurate value for $Q$ is estimated when $\alpha \neq 1$.

Fig. 3: Nyquist plots for experimental $Z_{\mathrm{g}, \exp }(\omega)$ and modeled $Z_{\mathrm{g}, \text { sim }}(\omega)$ electric impedance from $1 \mathrm{MHz}$ to $0.1 \mathrm{~Hz}$ with a voltage of $0.1 \mathrm{Vac}$, for different $\mathrm{KCl}$ concentrations in the hybrid micro nanochannel device. A. Electrical equivalent circuit proposed for the hybrid micro nanochannel device, where $Q, R$, and $R_{\mathrm{HF}}$ are attributed to the nanochannel non ideal capacitance, nanochannel resistance and microchannel resistance, respectively. B. Nanochannel filled with low $\mathrm{KCl}$ concentrations: $3 \times 10^{-6} \mathrm{M}(\mathrm{a}), 3 \times 10^{-5} \mathrm{M}(\mathrm{b}), 3 \times 10^{-4} \mathrm{M}(\mathrm{c})$, $3 \times 10^{-3} \mathrm{M}(\mathrm{d}), 3 \times 10^{-2} \mathrm{M}(\mathrm{e}) . \mathrm{C}$. Nanochannel with high $\mathrm{KCl}$ concentrations: $3 \times 10^{-1} \mathrm{M}(\mathrm{f})$, 3M (g). D. Measured and simulated conductances (left-axis: blue circles) across the nanochannel against the $\mathrm{KCl}$ ionic concentration. Two regimes appear in the log-log scale

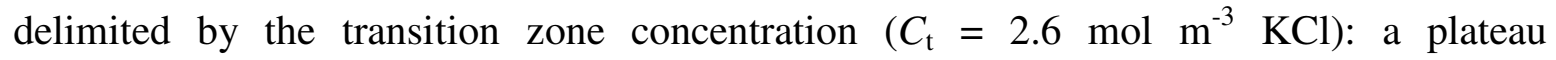
conductance for concentrations lower than $3 \times 10^{-2} \mathrm{~mol} \mathrm{~m}^{-3}$ and a linear conductance variation starting from concentrations higher than $30 \mathrm{~mol} \mathrm{~m}^{-3}$. The theoretical conductance 
curve change is calculated with equation 4 and the following parameters were found: effective surface charge density, $\sigma_{0}=-2.5 \times 10^{-2} \mathrm{C} \mathrm{m}^{-2}$, the nanochannel thickness, $h=170$ $\mathrm{nm}$, the average value of the ionic mobility $(\mathrm{KCl})$ and the positive $\left(\mathrm{K}^{+}\right)$charge mobility $\mu_{ \pm}$ $=7.765 \times 10^{-8} \mathrm{~m}^{2} \mathrm{~V}^{-1} \mathrm{~s}^{-1}$ and $\mu_{+}=7.62 \times 10^{-8} \mathrm{~m}^{2} \mathrm{~V}^{-1} \mathrm{~s}^{-1}$. Simulated capacitances (right-axis : green squares) across the nanochannel against the $\mathrm{KCl}$ ionic concentration. 


\section{Figures}

Figure 1

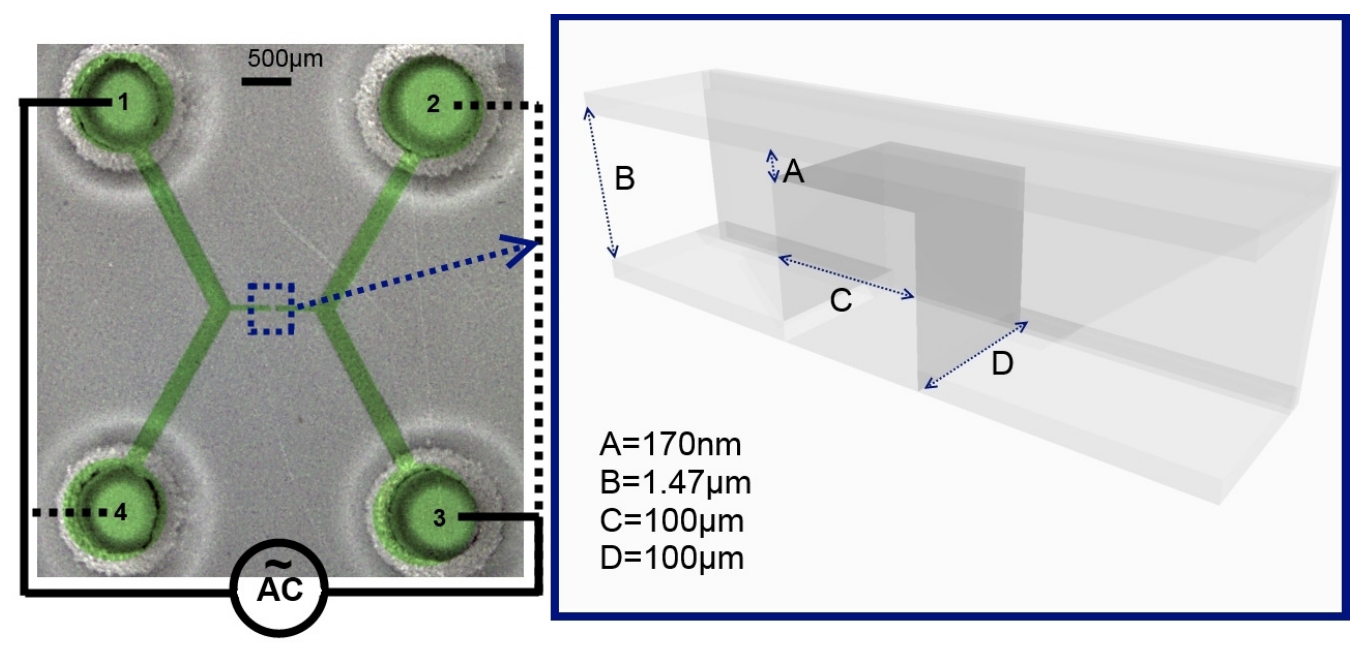


Figure 2
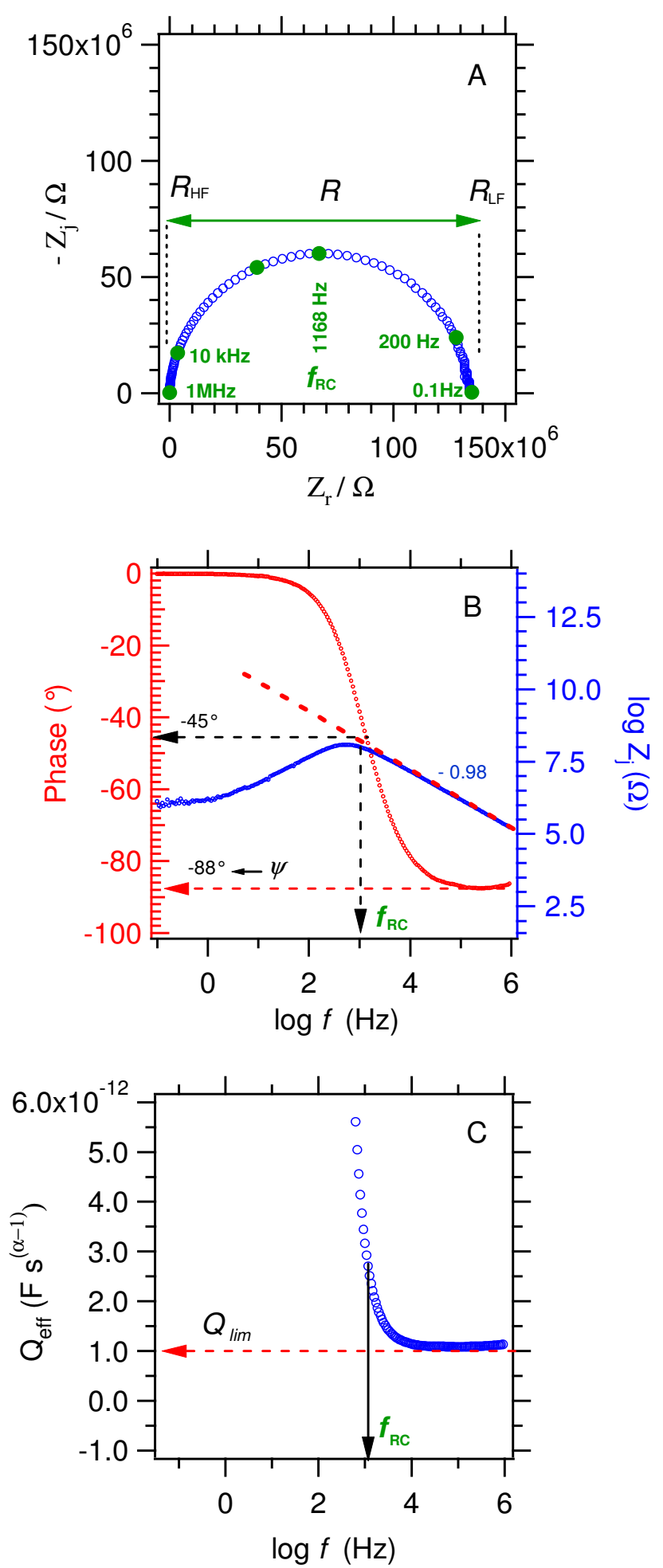
Figure 3

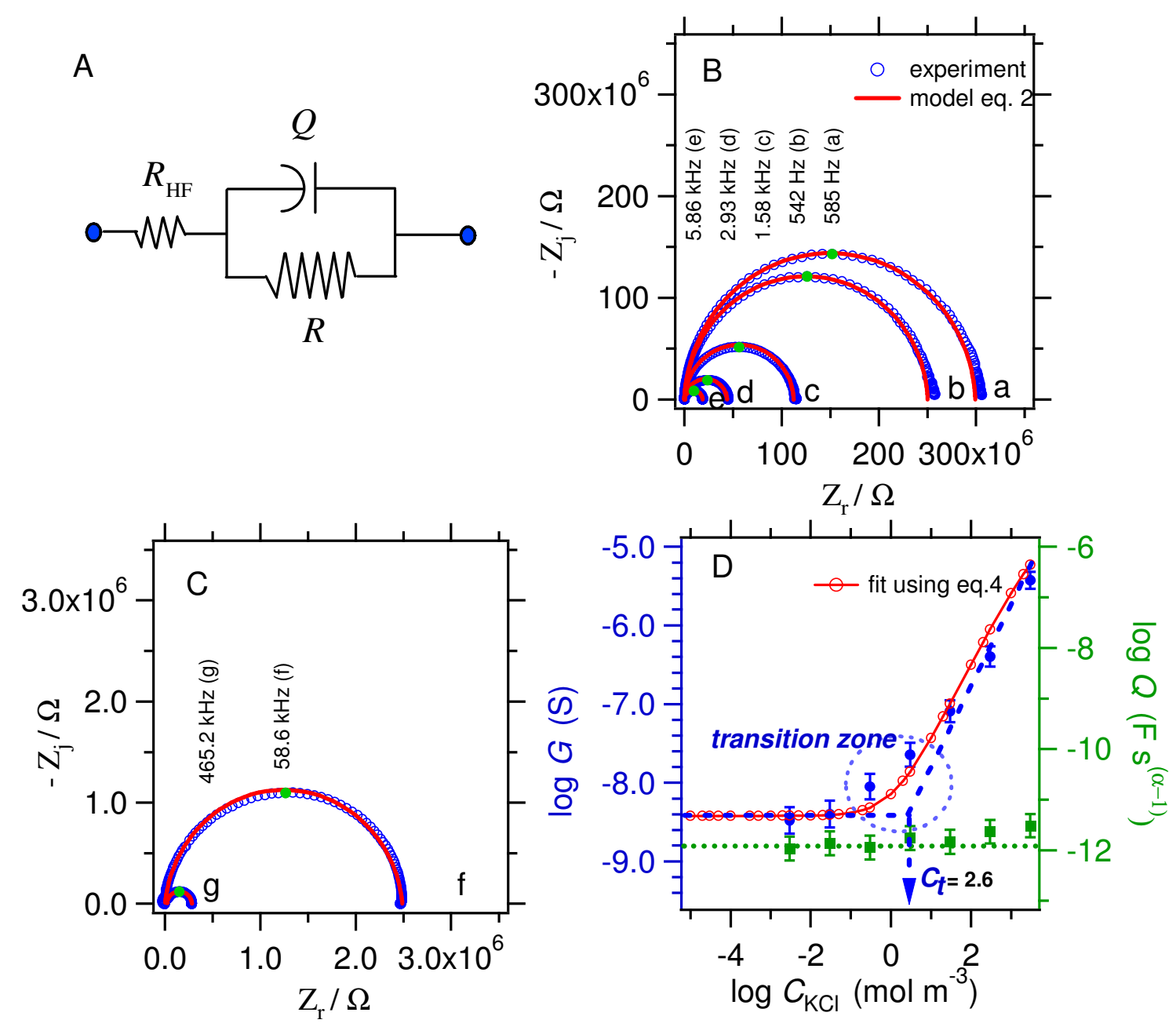

